\title{
Sampling Frequency Error Estimators for PRIME v1.4 systems
}

\author{
Javier Giménez*, José A. Cortés*, Alfredo Sanz ${ }^{\dagger}$, Luis Díez* \\ *Universidad de Málaga, E.T.S.I. Telecomunicación, Dpt. Ingeniería Comunicaciones, \\ Málaga (29010), Spain, email: \{javierg, jaca, diez\}@ic.uma.es \\ $\dagger$ Microchip Technology Inc., Av. de Juan Pablo II, 35, planta 7 \\ Zaragoza (50009), Spain, email: alfredo.sanz@microchip.com
}

\begin{abstract}
The performance of PRIME v1.4 systems might be severely degraded by a sampling frequency offset (SFO). This work addresses the problem of the SFO estimation by means of the pilots transmitted in the header and payload symbols. Estimators based on the best linear unbiased estimation principle are proposed and the frame error rate (FER) achieved with them is assessed both in an additive white Gaussian noise (AWGN) channel and in a set of 174 links obtained from measurements. Results indicate that the estimator based on the header symbols suffices to achieve almost the same performance of the no SFO case. Gains are shown to be particularly important when only the highest PRIME channel is employed and uncoded DQPSK and D8PSK are used.
\end{abstract}

Index Terms-sampling frequency offset, estimation, OFDM, PRIME.

\section{INTRODUCTION}

Smart metering is the most widespread Smart Grid application. It allows distribution system operators to remotely read the energy consumption and to perform basic operations on the meter such as connection/disconnection, among others. Narrowband power line communications (PLC) (NB-PLC) has proven to be a suitable technology for this purpose and is the most widely employed in Europe [1]. Among the commercial NB-PLC standards, the ones developed by the PRIME and G3-PLC alliances provide the highest bit rates.

This work focuses on the PRIME v1.4 specification [2], for which the work in [3] showed that performance degradation caused by an uncorrected sampling frequency offset (SFO) cannot be neglected, as it can be done in PRIME v1.3.6. The SFO causes three effects: the rotation of constellation symbols; the displacement of the discrete Fourier transform (DFT) window from its nominal position, causing intersymbol interference (ISI) and intersymbol intercarrier interference (ICI), and the intrasymbol ICI. The first effect is negligible for the maximum tolerance of the system clock $( \pm 50 \mathrm{ppm})$. For the second, a simple solution was proposed in [3]. However, while the frame error rate (FER) degradation caused by intrasymbol ICI was assessed in an additive white Gaussian noise (AWGN) channel for the coded modulations, no solution was proposed.

This paper extends the work in [3] by proposing a pilotbased strategy for estimating the SFO. It comprises two phases: a coarse estimation based on the header symbols, followed by a refined estimation performed over some of the payload symbols. The FER improvement reported by the proposed estimation method is assessed both in an AWGN channel and in a set of 174 links obtained from a measurement campaign accomplished in rural, semiurban and urban scenarios. Both coded and uncoded modulations are evaluated.

The rest of this paper is structured as follows. Section II describes the basic elements of PRIME v1.4, the channel model and summarizes the effects of the SFO on the received symbols. In section III, the proposed estimation procedure is described and its variance is assessed and compared to the Cramer-Rao bound (CRB). In section IV, the performance improvement given by the proposed estimators is evaluated. Finally, section V summarizes the main aspects of the paper.

\section{SYSTEM MODEL}

\section{A. PRIME v1.4 system}

PRIME v1.4 defines an orthogonal frequency division multiplexing (OFDM) system with 1024 carriers, generated using an inverse DFT (IDFT) with $N=2048$ points at a sampling frequency of $1 \mathrm{MHz}$. The cyclic prefix is $N_{g}=192$ samples, yielding an OFDM symbol length $N_{s}=N+N_{g}=2240$ samples (2.24 ms). Only 776 carriers, out of the 1024 ones, are employed. They are organized in 8 channels, with 97 carriers in each one. The referred channels can be independently used, being $\mathrm{N}_{\mathrm{ch}}$ the number that is actually transmitted.

As shown in [3], the normal transmission mode is the most sensitive to the SFO effects. Frames transmitted in normal modes, named type $A$, consist of an $N_{s}$-samples preamble followed by 2 header symbols and $M_{A}$ payload symbols, with $M_{A} \leq 63$. Differential in frequency modulations with DBPSK, DQPSK and D8PSK constellations are employed. A convolutional coder, with rate $1 / 2$ and a constraint length of 7 , can be optionally employed.

In order to perform the differential demodulation, a set of pilots are defined both for the header, $\mathcal{P}_{h}$, and payload symbols, $\mathcal{P}_{p}$. Header symbols have 13 equidistant pilot carriers per PRIME channel, while payload symbols have a single pilot carrier per PRIME channel, located at the beginning of the channel. Hence, $\left|\mathcal{P}_{h}\right|=13 \mathrm{~N}_{\mathrm{ch}}$ and $\left|\mathcal{P}_{p}\right|=\mathrm{N}_{\mathrm{ch}}$

The power spectral density (PSD) of the transmitted signal is fixed by the EN50065-1 for frequencies below $150 \mathrm{kHz}$. For the 150-500 kHz range, the unofficial limits proposed in the IEEE P1901.2 have been employed in this paper [4], [5]. 
The receiver used in this work for the convolutionally coded modulations consists of a differential demodulator that provides the unquantized log-likelihood ratio (LLR) values to a Viterbi decoder with a finite traceback length of 64 .

\section{B. Channel model}

Two different channel models are used in this work. First, the AWGN is employed to compare the variance of the proposed SFO estimators with the CRB and to draw qualitative conclusions about the factors that limit their performance. Second, a set of actual links obtained from a measurement campaign carried out in the rural, semiurban and urban scenarios described in [6] in the frequency band up to $500 \mathrm{kHz}$ is employed. While the number of measured links exceeds 100, a representative set of 29 channel responses and 6 noise signals have been selected for this study. These channel responses and noise signals are combined yielding a set of 174 links. Employed noise is modeled as a Gaussian stationary process with the PSD of the measured ones.

Fig. 1 depicts the signal to noise ratio (SNR) values corresponding to the employed links. Fig. 1 (a) depicts the average SNR of the eight channels $\mathrm{N}_{\mathrm{ch}}=8$, of the highest channel, $\left(\mathrm{N}_{\mathrm{ch}}=1, \mathrm{ch}=8\right)$, and of the lowest one, $\left(\mathrm{N}_{\mathrm{ch}}=1, \mathrm{ch}=1\right)$. As seen, the average SNR is generally higher in the lowest PRIME channel. While noise is known to be generally higher in the CENELEC A band than in the range above $350 \mathrm{kHz}$, this result is largely due to the transmitted PSD, which at 150 $\mathrm{kHz}$ and $500 \mathrm{kHz}$ is $21.1 \mathrm{~dB}$ and $31.1 \mathrm{~dB}$, respectively, lower than at $90 \mathrm{kHz}$.

Fig. 1 (b) depicts the minimum SNR values (which are the ones that limit the FER) in the lowest and highest PRIME channel. As a reference, the average SNR in the eight channels, $\mathrm{N}_{\mathrm{ch}}=8$, is also drawn. As seen, values are generally lower in $\mathrm{ch}=1$. This agrees with the known fact noise is more strongly colored in the CENELEC A band, because of the narrowband interference (NBI) and impulsive terms, than in the range above $350 \mathrm{kHz}$.

\section{Effect of the SFO}

Let's consider a PRIME v1.4 signal generated using a sampling period $T_{T X}$ and injected in a channel with additive stationary colored noise. When the received signal is sampled with period $T_{R X}$ at time instants $t=n T_{R X}+t_{0}$, with $0 \leq t_{0}<T_{R X}$, the output of the DFT for the $k$-th carrier of the $m$-th symbol can be written as (1). The terms $\Delta n=T_{R X} / T_{T X}-1$ and $n_{0}=t_{0} / T_{R X}$ denote the sampling period offset and sampling period phase error, respectively. For the sake of simplicity, $\Delta n$ will be referred to as SFO. $X_{k, m}$

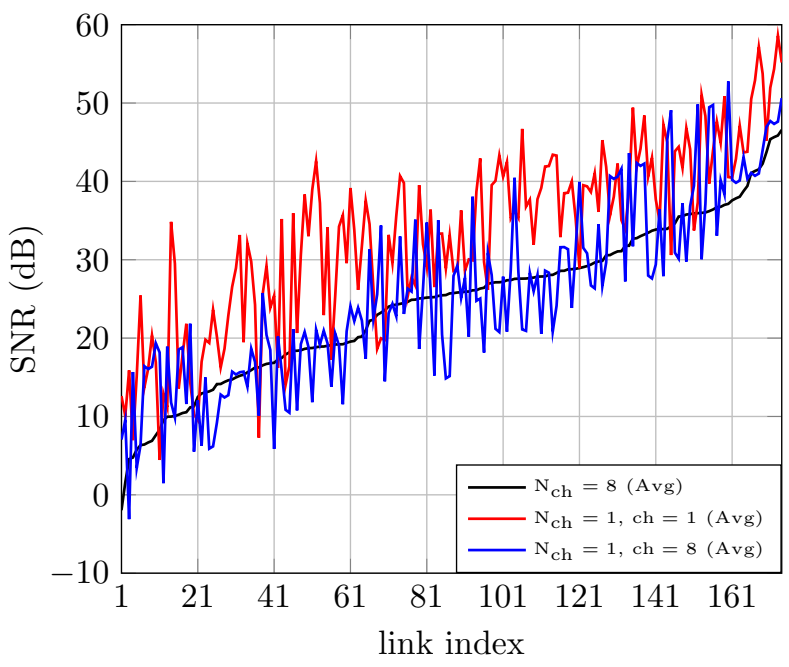

(a)

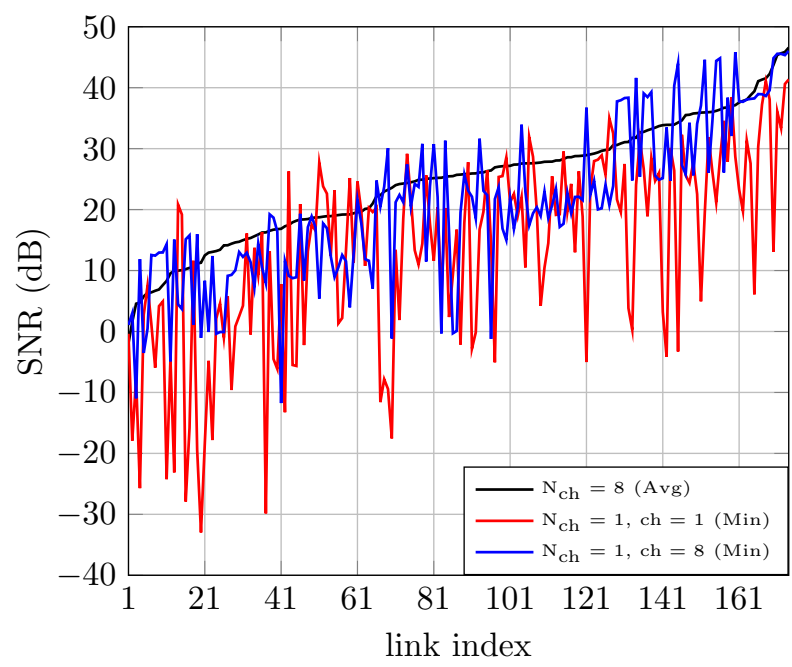

(b)

Fig. 1. (a) Average and (b) minimum SNR of the PRIME channels in 174 measured links.

denotes the complex value transmitted in the $k$-th carrier of the $m$-th symbol and $\ell_{m}=\left(d_{m}+\frac{N-1}{2}\right) \Delta n+n_{0}$, where $d_{m}$ represents the index of the first sample of the DFT window corresponding to the $m$-th symbol. Since the preamble lasts for $N$ samples, $d_{m}=m N_{s}+N_{g}+N$, where $m=0,1$ correspond to the header symbols and $m \geq 2$ to the payload ones.

As seen, the first term corresponds to the desired value, which is attenuated and rotated. The second term accounts for the contributions of the complex values transmitted in the

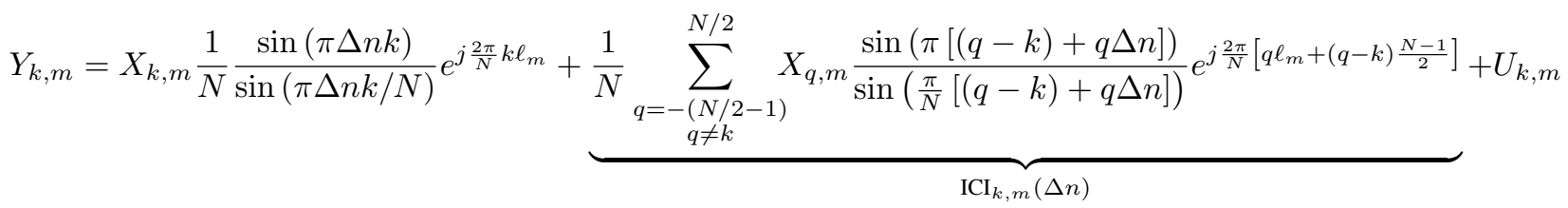


remaining carriers of the $m$-th symbol and will be referred to as intrasymbol ICI. The third term is a stationary Gaussian noise with power $\sigma_{k}^{2}$. Expression (1) has been derived assuming that the DFT only contains samples from the desired symbol, which can be achieved by using the strategy proposed in [3].

Neglecting the intrasymbol ICI and the attenuation of the desired value, the received value in (1) can be written as follows for $S N R_{k}=\mathbb{E}\left[\left|X_{k, m}\right|^{2}\right] / \sigma_{k}^{2} \gg 1$ [7]

$$
\begin{gathered}
Y_{k, m}=X_{k, m} e^{j \frac{2 \pi}{N} k\left[\left(d_{m}+(N-1) / 2\right) \Delta n+n_{0}\right]}+U_{k, m} \approx \\
X_{k, m} e^{j\left(\frac{2 \pi}{N} k\left[\left(d_{m}+(N-1) / 2\right) \Delta n+n_{0}\right]+\varphi_{k, m}\right)},
\end{gathered}
$$

where $\varphi_{k, m} \approx U_{k, m}^{\varphi} /\left|X_{k, m}\right|$, being $U_{k, m}^{\varphi} \sim \mathcal{N}\left(0, \sigma_{k}^{2} / 2\right)$ the component of the noise $U_{k, m}$ in the direction perpendicular to the signal term. Provided that all constellation symbols have equal amplitude, $\varphi_{k, m} \sim \mathcal{N}\left(0, \frac{1}{2 S N R_{k}}\right)$.

\section{SFO ESTIMATORS}

The method proposed to estimate $\Delta n$ consists of two phases. First, a coarse estimation is accomplished using the pilot tones transmitted in the header symbols. This estimate is assumed to be ideally corrected. The remaining SFO is then estimated using the pilots transmitted in the payload symbols. In order to assess the performance of the presented estimators, their variance will be compared to the CRB. The latter can be computed as [8, Sec. 3.7]

$$
\operatorname{var}(\widehat{\Delta n}) \geq\left[\boldsymbol{I}^{-1}(\boldsymbol{\theta})\right]_{11},
$$

where $\boldsymbol{I}(\boldsymbol{\theta})$ is the Fisher information matrix of the vector parameter $\boldsymbol{\theta}=\left[\Delta n, n_{0}\right]$, whose entries are given by

$$
[\boldsymbol{I}(\boldsymbol{\theta})]_{i j}=-\mathbb{E}\left[\frac{\partial \Lambda(\boldsymbol{Y} ; \boldsymbol{\theta})}{\partial \theta_{i}} \frac{\partial \Lambda(\boldsymbol{Y} ; \boldsymbol{\theta})}{\partial \theta_{j}}\right], \quad i, j=0,1,
$$

where $\mathcal{K}$ denotes the set of carriers used in the estimation, $\boldsymbol{Y}=\left[\boldsymbol{Y}_{m_{1}}, \ldots, \boldsymbol{Y}_{m_{M}}\right], \boldsymbol{Y}_{m}=\left[Y_{k_{1}, m}, \ldots, Y_{k_{|\mathcal{K}|}, m}\right]^{T},(\cdot)^{T}$ denotes the transpose operator, $\mathbb{E}[\cdot]$ is the expectation with respect to $\boldsymbol{Y}$ and $\Lambda(\boldsymbol{Y} ; \boldsymbol{\theta})$ denotes the $\log$-likelihood function given by

$$
\begin{aligned}
& \Lambda(\boldsymbol{Y} ; \boldsymbol{\theta})=-M|\mathcal{K}| \ln (\pi)-2 M \sum_{k \in \mathcal{K}} \ln \left(\sigma_{k}\right)- \\
& \sum_{m=m_{1}}^{m_{M}} \sum_{k \in \mathcal{K}} \frac{1}{\sigma_{k}^{2}}\left|Y_{k, m}-X_{k, m} e^{j \frac{2 \pi}{N}\left[\left(d_{m}+(N-1) / 2\right) \Delta n+n_{0}\right]}\right|^{2} .
\end{aligned}
$$

After some algebra, the CRB of $\widehat{\Delta n}$ can be written as

$$
\operatorname{var}(\widehat{\Delta n}) \geq \frac{N^{2}}{8 \pi\left[\sum_{m=m_{1}}^{m_{M}} d_{m}^{2}-\frac{1}{M}\left(\sum_{m=m_{1}}^{m_{M}} d_{m}\right)^{2}\right] \sum_{k \in \mathcal{K}} k^{2} S N R_{k}} .
$$

In order to obtain the CRB for the estimation based on the header symbols, expression (6) is particularized to $m_{1}=0$,
$m_{M}=1$ and $\mathcal{K}=\mathcal{P}_{h}$; and to $m_{1}=2, m_{M} \leq M_{A}+1$ and $\mathcal{K}=\mathcal{P}_{p}$ for the estimation based on the payload symbols.

\section{A. Coarse SFO estimation}

Assuming that the phase shift due to the SFO is small and denoting $Z_{k, m}=Y_{k, m} X_{k, m}^{*}$, the following approximation can be made for the header symbols

$$
\psi_{k}=\operatorname{Im}\left(Z_{k, 1} Z_{k, 0}^{*}\right) \approx \frac{2 \pi}{N} N_{s} k \Delta n+\underbrace{\varphi_{k, 1}-\varphi_{k, 0}}_{\Delta \varphi_{k}},
$$

for $k \in \mathcal{P}_{h}$ and where $\Delta \varphi_{k}$ is a zero-mean Gaussian noise with covariance matrix, $\mathbf{C}_{\Delta \varphi_{k}}=\frac{1}{2 S N R_{k}} \mathbf{I}_{\left|\mathcal{P}_{h}\right|}$, where $\mathbf{I}_{\left|\mathcal{P}_{h}\right|}$ is an identity matrix of rank $\left|\mathcal{P}_{h}\right|$.

The linearized model in (7) allows estimating $\Delta n$ by means of the best linear unbiased estimator (BLUE) [8, Sec. 6.3], which for this particular case can be expressed as

$$
\widehat{\Delta n}=\frac{N}{2 \pi N_{s}} \frac{\sum_{k \in \mathcal{P}_{h}} k S N R_{k} \operatorname{Im}\left(Z_{k, 1} Z_{k, 0}^{*}\right)}{\sum_{k \in \mathcal{P}_{h}} k^{2} S N R_{k}},
$$

and whose variance is given by

$$
\sigma_{\overline{\Delta n}}^{2}=\frac{N^{2}}{4 \pi^{2} N_{s}^{2} \sum_{k \in \mathcal{P}_{h}} k^{2} S N R_{k}} .
$$

Fig. 2 depicts the variance of (8) computed by means of simulations for $\Delta n=1 \mathrm{ppm}$ and $\Delta n=100 \mathrm{ppm}$, the variance in (9), referred to as theoretical, and the CRB. The simulated variance of the classical estimator in [9] is also depicted for comparison. For the sake of simplicity, $S N R_{k}=S N R$. As seen, the proposed estimator attains the CRB in almost the whole SNR range. On the contrary, while the estimator in [9] is also efficient for $\Delta n=1 \mathrm{ppm}$ (curves are indistinguishable), for $\Delta n=100 \mathrm{ppm}$ the proposed one outperforms it at a reduced computational complexity.

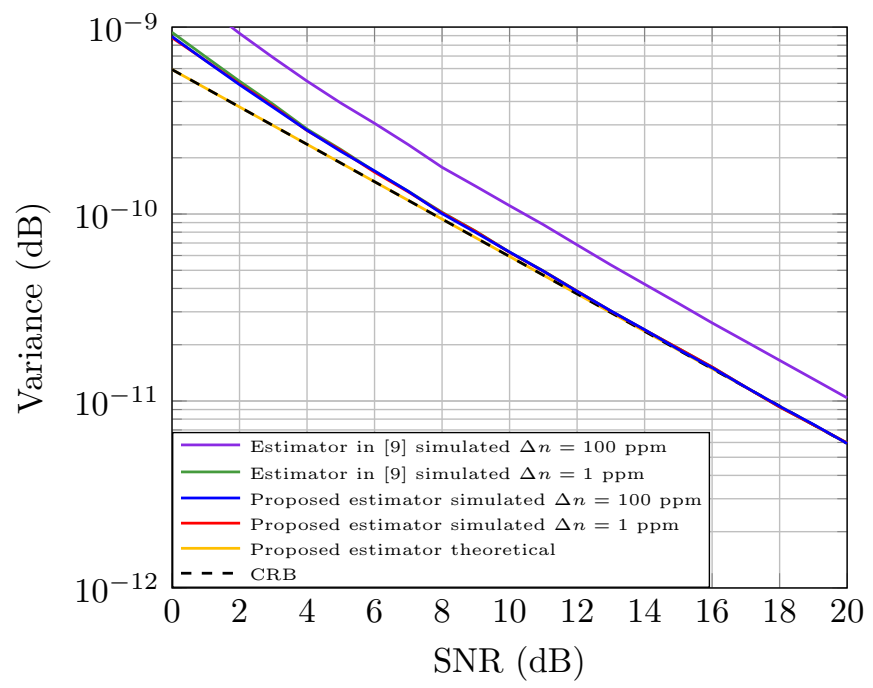

Fig. 2. CRB and variance of the coarse SFO estimator. 


\section{B. Fine SFO estimation}

The magnitude of the SFO to be estimated in the payload symbols, denoted as $\Delta n_{p}=\Delta n-\widehat{\Delta n}$, is notably lower than in the previous stage, since the SFO estimate obtained from the header symbols has already been corrected. The following approximation can then be also made

$$
\phi_{k}(m)=\operatorname{Im}\left(Z_{k, m} Z_{k, m-1}^{*}\right) \approx \underbrace{\frac{2 \pi}{N} N_{s} k \Delta n_{p}}_{\theta_{k}}+\Delta \varphi_{k}(m)
$$

where $\Delta \varphi_{k}(m)=\varphi_{k, m}-\varphi_{k, m-1}$ is a zero-mean Gaussian noise with a tridiagonal Toeplitz covariance matrix, $\mathbf{C}_{\Delta \varphi_{k}}$, with entries

$$
\left[\mathbf{C}_{\Delta \varphi_{k}}\right]_{i j}=\frac{1}{2 S N R_{k}} \begin{cases}2 & i=j \\ -1 & |i-j|=1 \\ 0 & \text { otherwise }\end{cases}
$$

Note that, for a given value of $k$, the estimation of $\theta_{k}$ from $M-1$ values of the observation $\phi_{k}(m)$ (obtained from $M$ OFDM symbols) can be accomplished using the BLUE, with the inverse of $\mathbf{C}_{\Delta \varphi_{k}}$ given in [10], yielding

$$
\widehat{\theta}_{k}=\frac{6}{(M-1) M(M+1)} \sum_{m=1}^{M-1} m(M-m) \phi_{k}(m),
$$

and whose variance is given by

$$
\sigma_{\widehat{\theta}_{k}}^{2}=\frac{6}{(M-1) M(M+1) S N R_{k}} .
$$

Since noise is uncorrelated among carriers, $\widehat{\theta}_{k}=\theta_{k}+\epsilon_{k}=$ $\frac{2 \pi}{N} N_{s} k \Delta n+\epsilon_{k}$, where $\epsilon_{k}$ is a zero-mean Gaussian noise with diagonal covariance matrix with entries $\left[\boldsymbol{C}_{\epsilon}\right]_{i i}=\sigma_{\widehat{\theta}_{i}}^{2}$, and $\Delta n$ can be estimated from the values of $\widehat{\theta}_{k}$ for $k \in \mathcal{P}_{p}$ using the BLUE, which in this case can be expressed as

$$
\widehat{\Delta n}_{p}=\frac{N}{2 \pi N_{s}} \frac{\sum_{k \in \mathcal{P}_{p}} k S N R_{k} \widehat{\theta}_{k}}{\sum_{k \in \mathcal{P}_{p}} k^{2} S N R_{k}},
$$

and whose variance is given by

$$
\sigma_{\overline{\Delta n}_{p}}^{2}=\frac{3 N^{2}}{2 \pi^{2} N_{s}^{2} M(M-1)(M+1)} \frac{1}{\sum_{k \in \mathcal{P}_{p}} k^{2} S N R_{k}} .
$$

Fig. 3 depicts the variance of (14) computed by means of simulations for $\Delta n_{p}=1 \mathrm{ppm}$ and $\Delta n_{p}=20 \mathrm{ppm}$, the variance in (15), referred to as theoretical, and the CRB for $M=2,10,20$. For the sake of simplicity, $S N R_{k}=S N R$. As seen, when $M=2$ the estimator is efficient in almost the whole SNR range. For $M=10$ and $M=20$ the estimator is no longer efficient but the performance loss with respect to the CRB has two different causes in the low and high SNR regimes. In the latter, the cause is that the errors in the approximation in (2) are no longer negligible with respect to the variance level. Hence, the larger the value of $M$, the larger the gap with respect to the CRB. In the low SNR regimen, the noise level is too large for the approximation $\operatorname{Im}\left(e^{j \beta}\right) \approx \beta$ to hold.

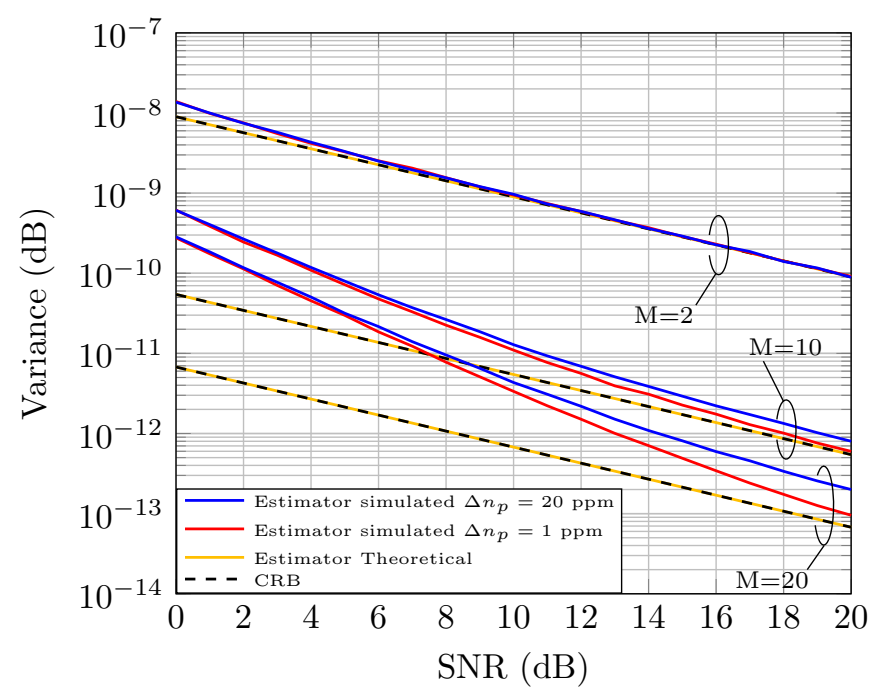

Fig. 3. CRB and variance of the fine SFO estimator.

\section{NUMERICAL RESULTS}

Assuming an ideal SFO correction system, the performance of the proposed estimators is assessed using two magnitudes: the signal to noise and distortion ratio (SNDR) gain and the FER. They are computed as follows. First, the ICI caused by a SFO $\widetilde{\Delta n}$ in the $k$-th carrier of the payload symbols is quantified by means of the signal to distortion ratio (SDR), which is defined as

$$
\operatorname{SDR}_{k}(\widetilde{\Delta n})=\frac{\sum_{m=2}^{M_{A}+1}\left|X_{k, m}\right|^{2}}{\sum_{m=2}^{M_{A}+1}\left|\mathrm{ICI}_{k, m}(\widetilde{\Delta n})\right|^{2}} .
$$

Then, the SNDR is obtained as

$$
\begin{aligned}
\operatorname{SNDR}_{k}(\widetilde{\Delta n}) & =\frac{\sum_{m=2}^{M_{A}+1}\left|X_{k, m}\right|^{2}}{\sum_{m=2}^{M_{A}+1}\left(\left|\mathrm{ICI}_{k, m}(\widetilde{\Delta n})\right|^{2}+\left|U_{k, m}\right|^{2}\right)} \\
& =\left(S N R_{k}^{-1}+S D R_{k}^{-1}\right)^{-1} .
\end{aligned}
$$

When no SFO correction is applied, $\widetilde{\Delta n}=\Delta n$ is deterministic, while when the SFO is estimated and corrected, $\widetilde{\Delta n}=\Delta n-\widehat{\Delta n}-\widehat{\Delta n}_{p}$ is a random variable. In the absence of SFO, $\widetilde{\Delta n}=0$. Hence, the correction of the SFO reports an average SNDR gain given by

$$
\Delta S N D R_{k}(\Delta n)=\mathbb{E}\left[S N D R_{k}(\widetilde{\Delta n})\right]-S N D R_{k}(\Delta n),
$$

where the expectation is performed over $\widetilde{\Delta n}$.

To avoid the time consuming simulations needed to obtain FER estimates, the following approximated procedure is employed. First, FER curves have been estimated in the absence 
of a SFO in an AWGN channel both for the coded an uncoded cases. These have been obtained assuming that frames are always detected and the header is decoded without errors. Hence, the FER is calculated only taking into account the payload symbols. Let's denote by $\mathcal{K}$ the overall set of carriers of the employed PRIME channels and by $\operatorname{FER}_{\mathrm{AWGN}}(S N R)$ the FER attained in an AWGN channel using a given constellation and coding scheme. The FER attained in a linear time-invariant (LTI) channel with Gaussian colored noise, SNR vector given by $S N R=\left\{S N R_{k}: k \in \mathcal{K}\right\}$, and SFO $\Delta n$, is here approximated as

$$
\operatorname{FER}(\boldsymbol{S N R})=\operatorname{FER}_{\mathrm{AWGN}}\left(\min _{k \in \mathcal{K}}\left\{\mathbb{E}\left[S N D R_{k}(\widetilde{\Delta n})\right]\right\}\right) .
$$

Throughout of this section, the maximum SFO experienced in PRIME systems, $\Delta n=100 \mathrm{ppm}$ is assumed.

\section{A. AWGN channel}

Fig. 4 depicts the minimum value of the SNDR in each PRIME channel when both the coarse and fine estimators are employed and when only the former is used. The fine estimator employs $M=10$. Estimation is performed over all PRIME channels. As expected, the higher the channel index, the higher the gain. Similarly, as the SNR increases, the ICI due to the SFO dominates and higher gains can be obtained by correcting it. As a reference, Fig. 4 also shows the SNR values required to receive the constellations that will be more affected by the SFO with a FER $=10^{-3}$. Significant gains can be obtained in the higher channels in the uncoded D8PSK and DQPSK. While gains are more modest in the coded D8PSK case, they are still considerable in $\mathrm{ch}=8$. The uncoded DBPSK has been omitted becase it requires almost the same SNR than the coded D8PSK but conveys only $1 \mathrm{bit} / \mathrm{symbol}$, while the latter conveys $3 / 2$ bits/symbol.

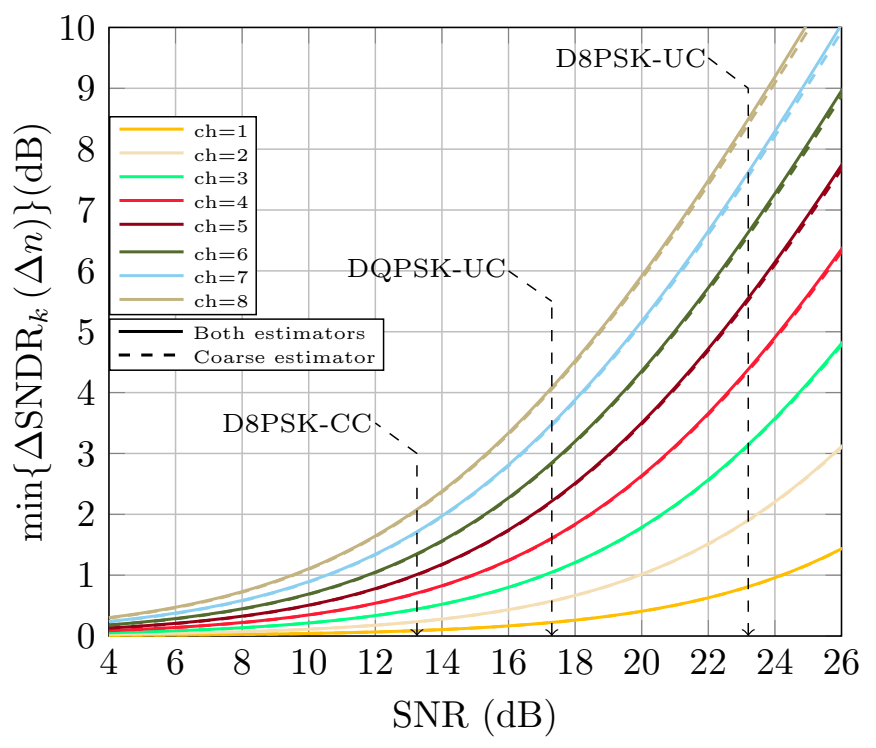

Fig. 4. Minimum SNDR gain in each PRIME channel.
It can be observed that using both estimators offers a very small gain with respect to using only the coarse one. However, it is interesting to note that Fig. 4 depicts average gains. Fig. 5 plots the probability density function (PDF) of the minimum SNDR gain in $\mathrm{ch}=8$ for the coded and uncoded D8PSK cases. In each case, the SNR is fixed to the value required to achieve FER $=10^{-3}$. As seen, using both estimators reduces the variance of the SNDR. Hence, actual need for the fine estimator has to be evaluated in terms of the FER.

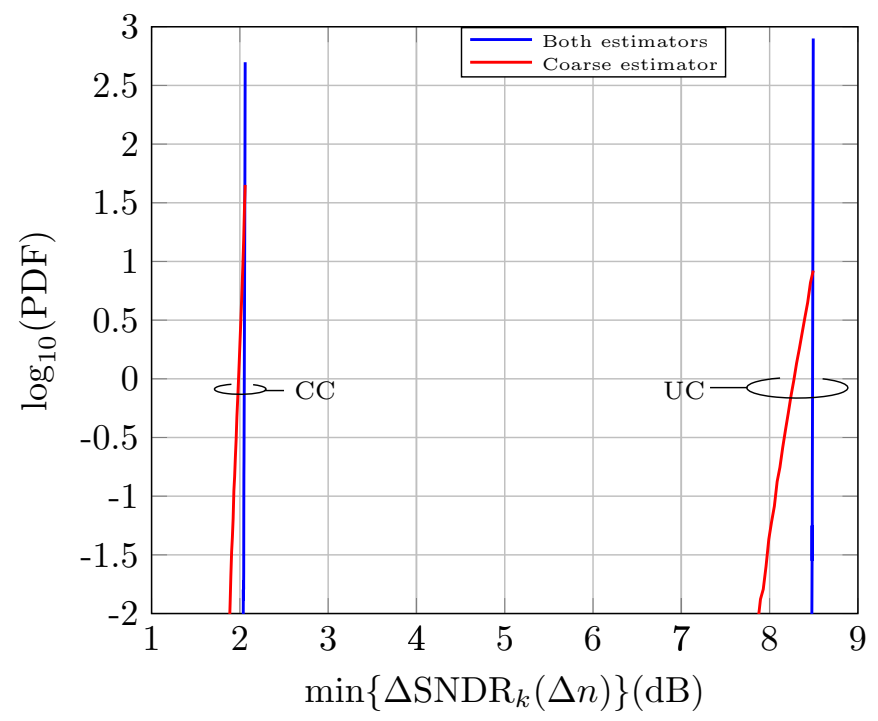

Fig. 5. Logarithm of the PDF of the minimum SNDR gain in $\mathrm{ch}=8$.

Fig. 6 depicts the FER for coded D8PSK and uncoded DQPSK and D8PSK. As seen, when the SFO is not corrected, the FER of coded D8PSK degrades $3 \mathrm{~dB}$ when $\mathrm{N}_{\mathrm{ch}}=1$, ch $=8$ and about $4 \mathrm{~dB}$ when $\mathrm{N}_{\mathrm{ch}}=8$, while for the uncoded D8PSK and DQSPK it becomes extremely degraded. When the proposed estimators are employed, the FER is almost equal to the ideal one $(\Delta n=0)$. Regarding the performance of the coarse estimator and the combined (coarse and fine) one, it can be noticed that former achieves almost the same performance than the latter for the coded D8PSK and uncoded DQPSK. Only for the uncoded D8PSK, the combined estimator performs slightly better $(0.25 \mathrm{~dB})$ than the coarse one when $\mathrm{N}_{\mathrm{ch}}=1$, ch $=8$. Hence, it can be concluded that the coarse estimator suffices for the modulations employed in PRIME v1.4.

It is worth mentioning that the worse FER of the case $\mathrm{N}_{\mathrm{ch}}=$ 8 with respect to $\mathrm{N}_{\mathrm{ch}}=1$, $\mathrm{ch}=8$ is due to the larger number of bits per frame of the former. Hence, while both cases have the same bit error rate (BER), since $\mathrm{N}_{\mathrm{ch}}=8$ has 8 times more bits than $\mathrm{N}_{\mathrm{ch}}=1$, ch $=8$, and a single bit error suffices for declaring the frame as erroneous, its resulting FER is larger.

\section{B. Actual channels}

Table I shows the percentage of the 174 actual links described in section II-B that support the considered constellation with a FER $\leq 10^{-3}$. Only the coarse estimator is employed. 


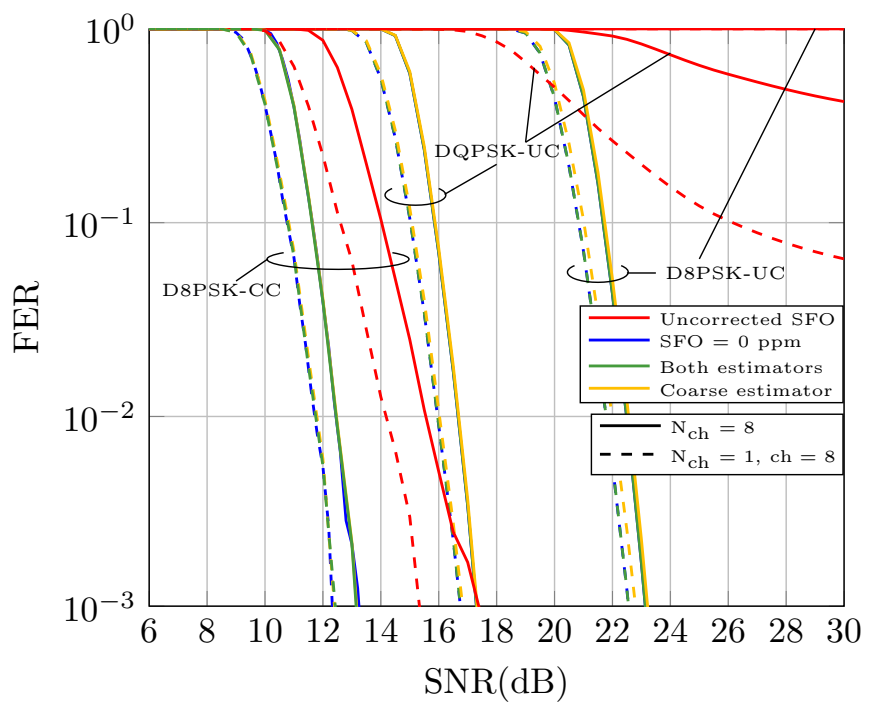

Fig. 6. FER in AWGN for different modulations with corrected and uncorrected $\mathrm{SFO}$.

As expected, no SFO correction would be needed when only the first PRIME channel is employed. On the contrary, when only the highest PRIME channel is employed $\left(\mathrm{N}_{\mathrm{ch}}=1\right.$, $\mathrm{ch}=8$ ), the uncoded DQPSK and D8PSK become unusable if the SFO is not corrected. In this case, the use of the proposed estimators reports significant gains, since the proportions of usable links rise up to $64.4 \%$ and $40.8 \%$, respectively. It is interesting to highlight that the latter value rises up to $41.4 \%$ (as in the no SFO case) if both estimators are employed. Gains reported by the proposed estimators when all PRIME channels are employed are more modest, but still interesting. It must be mentioned that, in this case, the proportion of usable links might be actually underestimated because of the procedure employed to estimate the FER, which is obtained from the minimum SNDR of all carriers.

TABLE I

Percentage (\%) OF ACTUAL LINKS WhICH SUPPORT CODED D8PSK AND UNCODED DQPSK/D8PSK WITH FER $\leq 10^{-3}$.

\begin{tabular}{ccccc} 
& & Coded & \multicolumn{2}{c}{ Uncoded } \\
\cline { 3 - 5 } & FER & D8PSK & DQPSK & D8PSK \\
\hline \multirow{2}{*}{$\mathrm{N}_{\mathrm{ch}}=8$} & SFO =0 ppm & 9.2 & 4.6 & 1.1 \\
& Corrected & 9.2 & 4.6 & 1.1 \\
& Uncorrected & 5.7 & 0.0 & 0.0 \\
\hline \multirow{2}{*}{$\mathrm{N}_{\mathrm{ch}}=1, \mathrm{ch}=8$} & SFO =0 ppm & 76.4 & 64.4 & 41.4 \\
& Corrected & 76.4 & 64.4 & 40.8 \\
& Uncorrected & 68.4 & 0.0 & 0.0 \\
\hline \multirow{2}{*}{$\mathrm{N}_{\mathrm{ch}}=1, \mathrm{ch}=1$} & SFO =0 ppm & 70.7 & 60.3 & 47.1 \\
& Corrected & 70.7 & 60.3 & 47.1 \\
& Uncorrected & 70.1 & 60.3 & 46.0 \\
\hline
\end{tabular}

\section{CONCLUSION}

This work has addressed the SFO estimation problem for PRIME v1.4 systems. A BLUE based on the pilots transmitted in the header symbols, refined by a BLUE based on the pilots transmitted on the payload symbols has been proposed. The FER improvement due to the use of the proposed estimators has been assessed in an AWGN channel and in a set of 174 measured links. It has been shown that the estimator based on the header symbols suffices to achieve almost the same performance of the case with no SFO. Larger gains have been obtained when only the highest PRIME channel is employed in links with high SNR. As an example, when the proposed method is employed, the proportion of assessed links where uncoded DQPSK and D8PSK can be used with FER $\leq 10^{-3}$ is $64.4 \%$ and $40.8 \%$, respectively. However, none of them can be if the SFO is uncorrected.

\section{ACKNOWLEDGMENT}

The authors thank Microchip Technology Inc. for the support provided to accomplish this work. The first author also thanks Universidad de Málaga for its financial support.

\section{REFERENCES}

[1] N. Uribe-Pérez, L. Hernández, D. de la Vega, and I. Angulo, "State of the Art and Trends Review of Smart Metering in Electricity Grids," Applied Sciences, vol. 6, no. 3, pp. 1-24, 2016.

[2] PRIME Alliance TWG, "Specification for PoweRline Intelligent Metering Evolution," 2014, Release 1.4.0.

[3] J. A. Cortés, A. Sanz, L. Díez, and F. J. Cañete, "Impact of Sampling Frequency Offset on PRIME 1.4 PLC Systems Performance," in Proceedings of the IEEE International Symposium on Power Line Communications and its Applications (ISPLC), 2017.

[4] European Committee for Electrotechnical Standardization (CENELEC), "Signalling on low-voltage electrical installations in the frequency range $3 \mathrm{kHz}$ to $148,5 \mathrm{kHz}$ Part 1: General requirements, frequency bands and electromagnetic disturbances," European Standard EN 50065-1, July 2001.

[5] IEEE P1901.2, "IEEE standard for low-frequency (less than $500 \mathrm{kHz}$ ) narrowband power line communications for Smart Grid applications," October 2013

[6] J. A. Cortés, A. Sanz, P. Estopiñán, and J. I. García, "Analysis of narrowband power line communication channels for advanced metering infrastructure," EURASIP Journal on Advances in Signal Processing, vol. 2015, no. 27, March 2015.

[7] S. A. Tretter, "Estimating the frequency of a noisy sinusoid by linear regression," IEEE Transactions on Information Theory, vol. IT-31, pp. 832-835, Nov. 1985.

[8] S. M. Kay, Fundamentals of Statistical Signal Processing: Estimation Theory. Prentice Hall, 1993.

[9] N. P. Sands and K. S. Jacobsen, "Pilotless Timing Recovery for Baseband Multicarrier Modulation," IEEE Journal on Selected Areas in Communications, vol. 20, no. 5, pp. 1047-1054, Jun. 2002.

[10] G. Meurant, "A Review on the Inverse of Symmetric Tridiagonal and Block Tridiagonal Matrices," SIAM Journal on Matrix Analysis and Applications, vol. 13, no. 3, pp. 707-728, Jul. 1992. 